\title{
TLR7 agonist induced repression of hepatocellular carcinoma via the TLR7-IKK-NF-кB-IL6 signaling pathway
}

\author{
$\mathrm{XINGBIN} \mathrm{REN}^{1}$, FEI WANG ${ }^{2}$, BAOJU JI $^{1}$ and $\mathrm{CHUNHAI} \mathrm{GAO}^{1}$ \\ Departments of ${ }^{1}$ Clinical Laboratory and ${ }^{2}$ Pain Management, Linyi People's Hospital, Linyi, Shandong 276003, P.R China
}

Received September 14, 2014; Accepted June 11, 2015

DOI: $10.3892 / 01.2016 .4329$

\begin{abstract}
Toll-like receptors (TLRs) are key members of innate immunity, involved in the defense against diseases, and evidence has revealed that TLR4/5 is involved in the carcinogenesis of hepatic cancer. TLR7 belongs to the TLR family, and its roles in immune-associated hepatic diseases have been well characterized; however, the consequences of agonist targeting of TLR7 in hepatic cancer have not previously been reported. The present study aimed to investigate the effects and underlying mechanisms of Imiquimod, a TLR7 agonist, on hepatic carcinogenesis by affecting the self-renewal of hepatic cancer stem cells. To detect the effects of this TLR7 agonist on hepatic cancer cells an MTT assay, mammosphere formation assay, ALDEFLUOR ${ }^{\mathrm{TM}}$ fluorescence-based stem cell sorting was used, and the potential signaling involved in the mechanism was investigated by western blot analysis. The TLR7 agonist Imiquimod demonstrated inhibitory effects on the cell proliferation and mammosphere formation of hepatic cells and stem cells, and decreased stem cell number $(\mathrm{P}<0.01)$. These effects may be achieved via the TLR7/I $\kappa \mathrm{B}$ kinase/nuclear factor- $\kappa \mathrm{B} /$ interleukin- 6 signaling pathway, with decreased levels of Snail expression. The present study demonstrated the effects and mechanisms of the TLR7 agonist on hepatic cancer occurred via suppression of the self-renewal of cancer stem cells, indicating novel potential functions of the TLR7 agonist in the treatment of HCC.
\end{abstract}

\section{Introduction}

Worldwide, hepatocellular carcinoma (HCC) is one of the most aggressive types of malignancy $(1,2)$. Due to its poor prognosis, $\mathrm{HCC}$ is the third leading cause of cancer-associated mortality worldwide, a statistic which is even higher in China (3). There is a marked geographical variation in the incidence of HCC,

Correspondence to: Professor Chunhai Gao, Department of Clinical Laboratory, Linyi People's Hospital, 27 Jiefang Road, Linyi, Shandong 276003, P.R China

E-mail: prof_chunhai_gao@163.com

Key words: hepatic carcinoma, toll-like receptors, Toll-like receptor 7 ligand, Imiquimod, cancer stem cells and $>80 \%$ of cases occur following hepatitis infection. Furthermore, HCC is typically more severe in China, with a poorer 5-year survival compared with Western countries (4). The tumorigenesis of HCC is a multistage process, and it has been reported that aberrant Toll-like receptor (TLR) signaling may contribute to the development and progression of HCC. TLRs are pattern recognition receptors, which have significant functions in host defense via recognition of pathogen-associated molecular patterns (5). Studies have suggested that TLR signaling is involved in the progression of certain chronic liver diseases. In addition, ongoing clinical trials have suggested that therapeutic manipulation of TLR pathways may represent a novel strategy for the reversal of chronic liver disease (6). Following activation by their respective ligands, TLRs initiate intracellular pro-inflammatory/anti-inflammatory signaling cascades through the recruitment of various adaptor proteins. TLR-associated signaling pathways are therefore tightly regulated to prevent inappropriate generation of pro-inflammatory cytokines and interferons, and thereby prevent aberrant autoimmune and inflammatory processes.

Evidence has indicated that TLR4/5 are involved in the carcinogenesis of hepatic cancer, indicating that anti-TRLs are useful and applicable in this disease entity $(7,8)$. In normal liver tissues, TLRs have crucial roles in the processes of wound healing and regeneration. In addition, TLRs are involved in the pathogenesis and progression of various inflammatory liver diseases, including autoimmune liver disease, non-alcoholic steatohepatitis, alcoholic liver disease, fibrogenesis and chronic hepatitis $\mathrm{B} / \mathrm{C}$ virus $(\mathrm{HBV} / \mathrm{HCV}$ ) infection (9). Further studies have suggested that TLR-based therapies may represent a promising strategy for the treatment of viral hepatitis. Since hepatitis is closely associated with liver cirrhosis and further HCC, and the signaling that was observed to function in hepatitis also functions in cancer biology $(10,11)$, it was hypothesized that TLR-based therapies may be useful therapeutic strategies for the malignancies associated with hepatic tumors. Our preliminary experiments revealed that TLR7 is expressed in HCCs, therefore, the effects of treatment with a TLR7 agonist were investigated in the present study.

In human tissues, TLR7 is only expressed on human plasmacytoid dendritic cells, which TLR7 agonist Imiquimod was able to bind (12). Imiquimod has been demonstrated to be a strong inducer of interferon $\gamma$ and a wide range of 
pro-inflammatory mediators $(13,14)$. Immune system reactions and disorders are regarded as critical processes in tumor development and initiation; however, the mechanism underlying the effects of the TLR7 agonist in regulating cancer initiation and development has remained to be elucidated. Cancer stem cells (CSCs) were regarded as the roots of tumor recurrence, and also contribute to chemoradiotherapy resistance $(15,16)$. TAM tyrosine kinases regulate the targeted genes involved in the homeostatic regulation of cytokine receptors and TLR-mediated signal transduction pathways. A loss of TAM receptors, attributed to exaggerated inflammatory responses by microglia and characterized by increased mitogen-activated protein kinase, IкB kinase (IKK) and nuclear factor (NF)- $\mathrm{kB}$ activation, as well as elevated production of pro-inflammatory cytokines, is detrimental to stem cell proliferation (10). IKK, NF- $\mathrm{KB}$ and interleukin-6 (IL6) signaling have previously been demonstrated to be involved in the regulation of cancer stem cells; therefore, the present study aimed to elucidate whether the TLR7 agonist exhibited suppressive effects on hepatic cancer cells, potentially through IKK and NF- $\kappa B$ activation.

\section{Materials and methods}

Cell culture. The HCCLM3 and MHCC97-H human HCC cell lines were purchased from the American Type Culture Collection (Manassas, VA, USA) and cultured in Dulbecco's modified Eagle's medium (DMEM; Invitrogen, Carlsbad, CA, USA), containing $10 \%$ fetal bovine serum (FBS) and $1 \%$ penicillin and streptomycin (Invitrogen). Mammospheres (CSCs) obtained from the HCCLM3 and MHCC97-H cell lines were cultured in DMEM/Ham's F-12 medium, supplemented with $10 \mathrm{ng} / \mathrm{ml}$ epidermal growth factor, $10 \mathrm{ng} / \mathrm{ml}$ human basic fibroblast growth factor, $1 \mu \mathrm{g} / \mathrm{ml}$ hydrocortisone, $4 \mu \mathrm{g} / \mathrm{ml}$ insulin and $1 \%$ penicillin and streptomycin (Invitrogen) (17). All cells were cultured in $5 \% \mathrm{CO}_{2}$ at $37^{\circ} \mathrm{C}$.

Western blot analysis. Proteins were harvested from the HCC cells after $48 \mathrm{~h}$ with radioimmunoprecipitation assay lysis buffer (BioTeke Corp., Beijing, China) and $60 \mu \mathrm{g}$ cellular protein was subjected to $10 \%$ SDS-PAGE separation. Proteins were transferred to polyvinylidene difluoride microporous membranes (EMD Millipore, Billerica, MA, USA) and blots were probed with rabbit monoclonal IL6 antibody (1:2500; ab32530; Abcam, Cambridge, MA, USA), internal control GAPDH (1:5000; sc-25778), mouse monoclonal immunoglobulin $\mathrm{G}(\operatorname{IgG})_{2 \mathrm{a}}$ IKK antibody (1:1000; sc-376114) and rabbit polyclonal IgG NF- $\mathrm{KB}$ antibody (1:1000; sc-372) (Santa Cruz Biotechnology, Inc., Dallas, TX, USA). All primary antibodies were incubated overnight at $4^{\circ} \mathrm{C}$.

Cell proliferation assay. Imiquimod [1-(2-methylproph yl)-1H-imadazo(4,5c)quinoline-4-amine] is a synthetic low-molecular-weight compound, which is a topical immune response modifier that upregulates immune responses. Imiquimod (catalog no. R837; InvivoGen, San Diego, CA, USA) and TLR7 ligand (cat. no. ODN 20959; Miltenyi Biotec Inc., Auburn, CA, USA) were used. The cells of each group were treated with $100 \mathrm{nM}, 1 \mu \mathrm{M}, 10 \mu \mathrm{M}, 100 \mu \mathrm{M}$ and $1 \mathrm{mM}$ Imiquimod and/or $10 \mathrm{nM}, 100 \mathrm{nM}, 1 \mu \mathrm{M}$ and $10 \mu \mathrm{M}$ TLR7 ligand for 24,48 or $72 \mathrm{~h}$ prior to MTT detection. The MTT (Sigma-Aldrich, St Louis, MO, USA) spectrophotometric dye assay was used to detect cell proliferation ability. HCCLM3 and MHCC97-H cells were plated in 96-well plates at a density of 2000 cells/well, respectively. At 24 , 48 and $72 \mathrm{~h}$ post-treatment, cells were incubated with $10 \mu \mathrm{l}$ MTT for $4 \mathrm{~h}$. The color was developed by incubating the cells with $100 \mu \mathrm{l}$ dimethyl sulfoxide; and the absorbance was detected at a wavelength of $490 \mathrm{~nm}$ using a Bio-Rad 550 Ultramark $^{\mathrm{TM}}$ Microplate Reader (Bio-Rad Laboratories, Inc. Hercules, CA, USA). The data were obtained from three independent experiments.

Sphere formation assays. To explore the effects of Imiquimod and TRL7 ligand on the self-renewal of HCC cells, cells of the various groups were plated in ultra-low attachment (non-adherent condition) dishes (Corning, Inc., Corning, NY, USA) to test their ability to form primary mammospheres when incubated at $37^{\circ} \mathrm{C}$. On day 7 , cell mammosphere numbers were counted under the low-power field of an inverted microscope (DMI3000B; Leica Microsystems, Wetzlar, Germany); briefly, the mammospheres were collected by centrifugation at $450 \mathrm{x} \mathrm{g}$ for $5 \mathrm{~min}$ at $4^{\circ} \mathrm{C}$, and dissociated mechanically with a pipette using $0.05 \%$ Trypsin-EDTA (Invitrogen). The obtained spheres were passed through a $40-\mu \mathrm{m}$ sieve and analyzed microscopically for single cellularity. The mammosphere forming efficiency (MFE) was calculated as the percentage ratio between the obtained spheres and plated cells [mean \pm standard deviation (SD) of three independent experiments] (18). Obtained mammospheres of the various groups were disaggregated into single cells, and these cells were re-suspended and plated to test the ability of self-renewal of the second generation for $\leq 7$ days. The number of secondary mammospheres was also calculated as the mean $\pm \mathrm{SD}$.

Immunofluorescence assay. The HCCLM3 and MHCC97-H cells $\left(1-3 \times 10^{4} /\right.$ well $)$ were plated in a chamber $20 \mathrm{~h}$ prior to staining, then fixed in $10 \%$ formalin (Thermo Fisher Scientific, Inc., Waltham, MA, USA) for $15 \mathrm{~min}$. The cells were blocked with 5\% goat serum (cat. no. ab7481; Abcam), and incubated with the primary monoclonal rabbit anti-Snail antibody (1:500; cat. no. 3879; Cell Signaling Technology, Inc., Danvers, MA, USA) overnight at $4^{\circ} \mathrm{C}$. The membranes were then washed three times with phosphate-buffered saline (PBS; Calbiotech Inc., Spring Valley, CA, USA) with Tween-20 (Thermo Fisher Scientific, Inc.) (PBST) for $15 \mathrm{~min}$. The membranes were then incubated with a horseradish peroxidase-conjugated goat anti-mouse IgG secondary antibody (cat. no. sc-2060; 1:,5000; Santa Cruz Biotechnology, Inc.) for $1 \mathrm{~h}$ at room temperature and washed with PBST. Next, the membranes were incubated with Alexa Fluor ${ }^{\circledR} 488$ secondary antibody (goat IgG; cat. no. Z25002; 1:1,000; Invitrogen) for $1 \mathrm{~h}$ at room temperature. Next, the membranes were incubated with DAPI (Thermo Fisher Scientific, Inc.) for $10 \mathrm{~min}$, then washed in PBS. Fluorescence was visualized using a Leica microscope (DMI3000B; Leica Microsystems).

Flow cytometric analysis. To further confirm the influence of Imiquimod and the TLR7 ligand on the self-renewal ability of stem cells the ALDEFLUOR ${ }^{\mathrm{TM}}$ fluorescent reagent system 
A
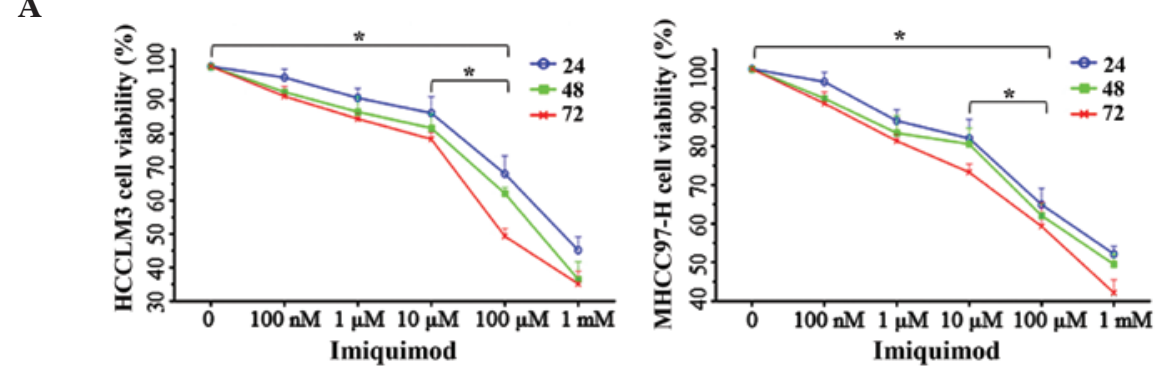

B
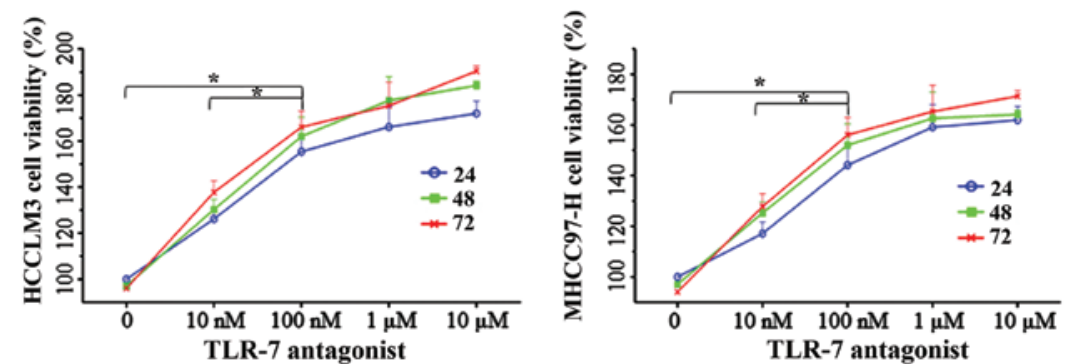

C

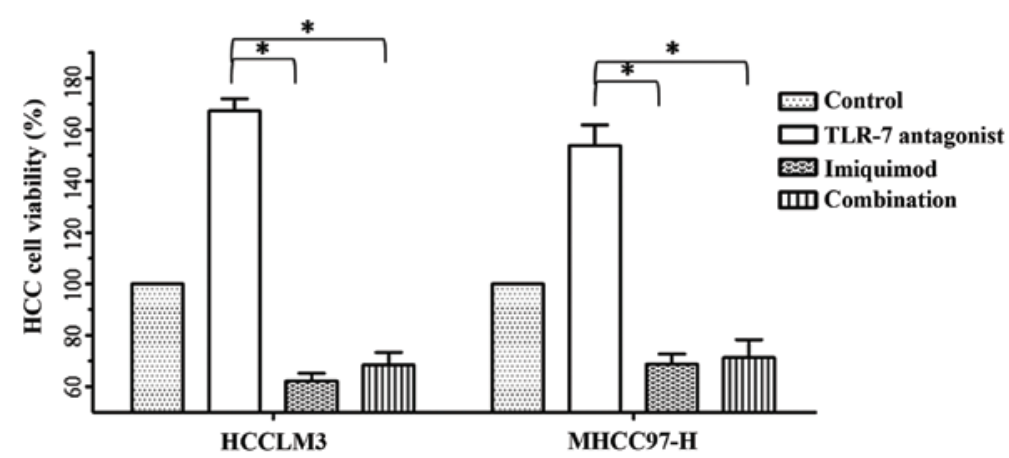

Figure 1. Effects of Imiquimod and TLR7 ligand alone or in combination on HCC cell proliferation were detected by MTT assay. (A) Imiquimod (100 $\mu$ M) was demonstrated to exert effective repression on cell proliferation of the HCCLM3 and MHCC97-H HCC cell lines following $48 \mathrm{~h}$ of incubation, $\mathrm{P}<0.01$. (B) TLR7 ligand (100 nM) significantly induced cell proliferation, ${ }^{*} \mathrm{P}<0.01$. (C) A dose of $100 \mu \mathrm{M}$ Imiquimod effectively reversed the oncogenic effect of the TLR7 ligand, compared with each of the drugs alone at $48 \mathrm{~h}$ "P<0.01. HCC, hepatocellular carcinoma; TLR7, Toll-like receptor 7.

(STEMCELL Technologies, Inc., Vancouver, Canada) was used to separate aldehyde dehydrogenase $(\mathrm{ALDH})^{+}$cells from the various groups, as previously described (15). Flow cytometer setup and data acquisition were followed by ALDH-based Cell Detection kit analysis (ALDEFLUOR).

Statistical analysis. Data are expressed as the mean \pm SD and were analyzed by Student's t-test and $\chi^{2}$ test, using SPSS for Windows version 16.0 (SPSS, Inc., Chicago, IL, USA).

\section{Results}

The functions of TLR7 signaling in HCC cell proliferation. To identify the effects of the TLR7 signaling pathway on the proliferative potential of $\mathrm{HCC}$ cells, the cells were treated with TLR7 ligand and/or Imiquimod. Cell proliferation was evaluated using the MTT method at 24, 48 and $72 \mathrm{~h}$, and the proliferation of cancer cells treated with $100 \mu \mathrm{M}$ Imiquimod for $48 \mathrm{~h}$ was suppressed most effectively, compared with the other groups ( $\mathrm{P}<0.01$; Fig. 1A). Conversely, $100 \mathrm{nM}$ TLR7 ligand significantly induced cell proliferation (Fig. 1B); however, the induction of proliferation was blocked by the addition of $100 \mu \mathrm{M}$ Imiquimod, indicating that $100 \mu \mathrm{M}$ Imiquimod effectively reversed the oncogenic function of the TLR7 ligand following $48 \mathrm{~h}$ of incubation (Fig. 1C).

Imiquimod suppresses mammosphere formation ability of HCC cells. The MFE of Imiquimod-treated HCCLM3 (HCCLM3-I) cells $(0.92 \pm 0.11 \%)$ was significantly lower than that of the HCCLM3 control group $(2.54 \pm 0.28 \%)$. In addition, the MFE of Imiqumod-treated MHCC97 (MHCC97-H-I) cells $(1.14 \pm 0.104 \%)$ was markedly lower than that of the MHCC97-H control group $(3.32 \pm 0.25 \%)(\mathrm{P}<0.01 ;$ Fig. $2 \mathrm{~A}$ and $\mathrm{B})$. Disaggregation of primary mammospheres and secondary plating of suspended cells resulted in the repeated formation of mammospheres, which suggested that the mammospheres of each group in the first round contained cells capable of self-renewal. The MFE of HCCLM3-I secondary mammospheres $(1.37 \pm 0.22 \%)$ was lower than that of the HCCLM3 control group $(4.21 \pm 0.27 \%)$; and the MFE of MHCC 97-H-I secondary mammospheres $(1.98 \pm 0.16 \%)$ was lower than that of the MHCC97-H control group $(4.76 \pm 0.53 \%)$ $(\mathrm{P}<0.01$; Fig. 2C and D). To further confirm the suppressive effects of Imiquimod on $\mathrm{HCC}$ stem cells, the proportion of $\mathrm{ALDH}^{+}$cells was detected in the various groups, and Imiquimod was demonstrated to significantly inhibit CSC numbers ( $\mathrm{P}<0.01$; Fig. 3A).

Imiquimod reverses TLR7 ligand-induced mammosphere formation ability. The MFE of TLR7 ligand-treated HCCLM3 


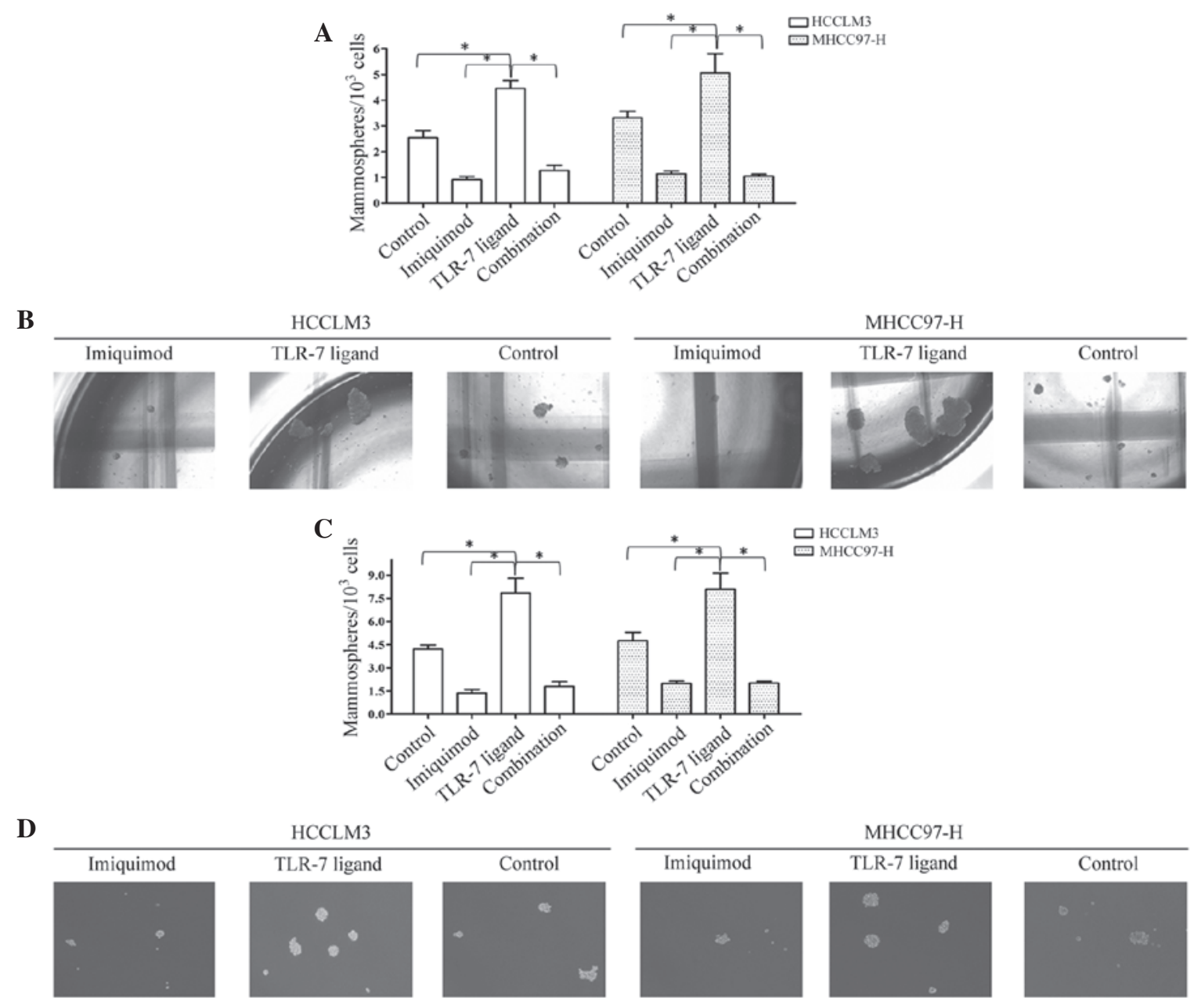

Figure 2. Suppressive function of Imiquimod on the self-renewal of HCC cells. HCC cells were seeded in ultra-low attachment plates to facilitate formation of the $1^{\text {st }}$ generation of mammospheres. Subsequently, the cells forming mammospheres were reseeded to acquire the $2^{\text {nd }}$ generation of mammospheres. (A) $1^{\text {st }}$ generation MFE of various treatment groups of the HCCLM3 and MHCC97-H HCC cell lines, ${ }^{*} \mathrm{P}<0.01$. (B) Representative image of the 1st generation mammospheres of the various groups. (C) $2^{\text {nd }}$ generation MFE of various treatment groups of the HCCLM3 and MHCC97-H HCC cell lines, "P $<0.01$. (D) Representative images of the $2^{\text {nd }}$ generation of mammospheres of the two HCC cell lines. Imiquimod effectively inhibited the MFE regardless of the TLR7 ligand. HCC, hepatocellular carcinnoma; MFE, mammosphere forming efficiency; TLR7, Toll-like receptor 7.

(HCCLM3-L) cells $(4.46 \pm 0.31 \%)$ was elevated significantly, compared with that of the control group $(2.54 \pm 0.28 \%)(\mathrm{P}<0.01)$. In addition, the MFE of TLR7 ligand-treated MHCC97-H (MHCC97-H-L) cells $(5.06 \pm 0.75 \%)$ was markedly higher than that of the MHCC $97-\mathrm{H}$ control group $(3.32 \pm 0.25 \%)$, $\mathrm{P}<0.01$ (Fig. 2A and B). The MFE of HCCLM3-L secondary mammospheres was $7.85 \pm 0.94 \%$, compared with $4.21 \pm 0.27 \%$ of the HCCLM3 control group $(\mathrm{P}<0.01)$; while the MFE of MHCC97-H-L secondary mammospheres was higher $(8.07 \pm 1.06 \%)$ than that of the MHCC97-H control group $(4.76 \pm 0.53 \%)(\mathrm{P}<0.01$; Fig. $2 \mathrm{C}$ and D). Furthermore, the TLR7 ligand enhanced the number of $\mathrm{ALDH}^{+}$cells of $\mathrm{HCC}$, while Imiquimod abrogated the TLR7 ligand-induced increase in mammosphere numbers (Fig. 2) and inhibited the $\mathrm{ALDH}^{+}$ cells $(\mathrm{P}<0.01)$. Combined treatment with Imiquimod and TLR7 ligand therefore neutralized the oncogenic function of the TLR7 ligand in the self-renewal of CSCs ( $\mathrm{P}<0.01$; Fig. 2).

Imiquimod inhibits HCC cells and CSCs via the IKK-NFKB-IL6 signaling pathway. To explore the potential mechanisms by which Imiquimod suppresses CSC numbers, it was hypothesized that
Imiquimod inhibited malignant cellular behaviors in HCC cells and HCC stem cells through downregulation of IKK-NFKB-IL6 signaling, which had previously been demonstrated to function in TLR7-dominated hepatic diseases $(8,9,19)$. The expression levels of IKK, NFKB and IL6 were all significantly decreased in the Imiquimod-treated group $(\mathrm{P}<0.01$; Fig. $3 \mathrm{~B})$; and were identical in the TLR7 ligand and Imiquimod combined group (data not shown). To further confirm the suppressive effects of Imiquimod on stem cells, Snail, which is an EMT marker, identifying stem cell pluripotency, was detected in HCC cells using immunofluorescent staining. The results indicated that Snail expression was significantly inhibited in Imiquimod-treated HCC cells (Fig. 3C) and HCC cancer stem cells, compared with that of the control group (Fig. 3C). The results also indicated that the Snail signal was markedly stronger in stem cells than that in differentiated cancer cells.

\section{Discussion}

Liver cancer is one of the leading causes of cancer-associated mortality worldwide, and therefore the improvement of 
A

HCCLM3
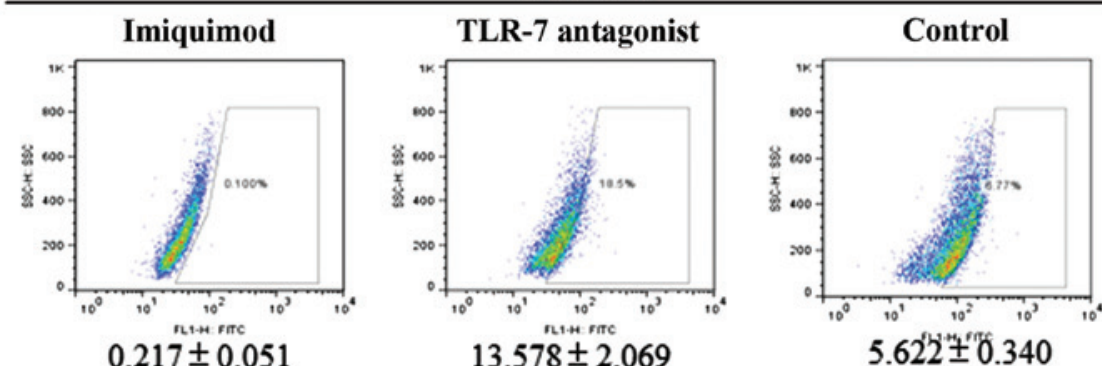

MHCC97-H

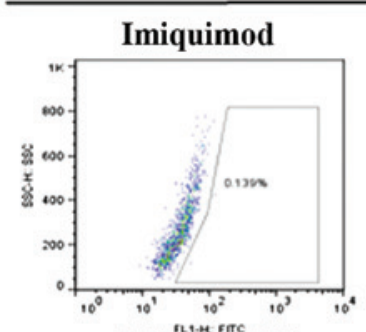

$0.335 \pm 0.034$
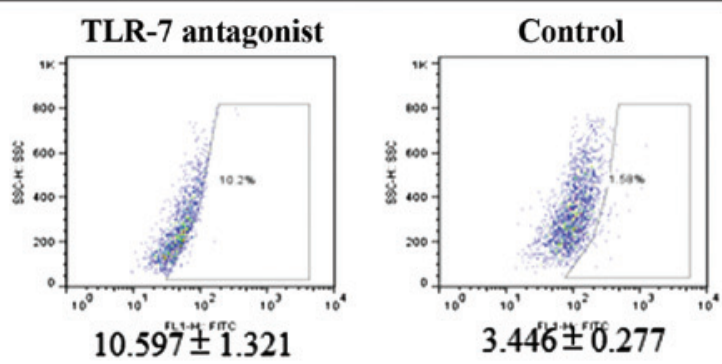

B

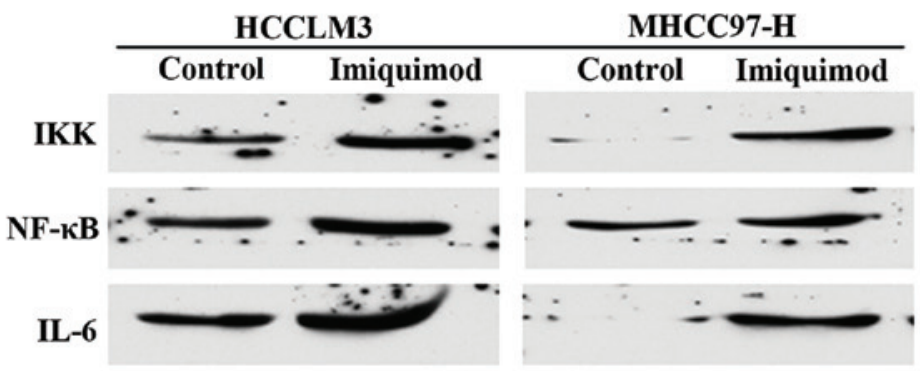

C

HCC cells

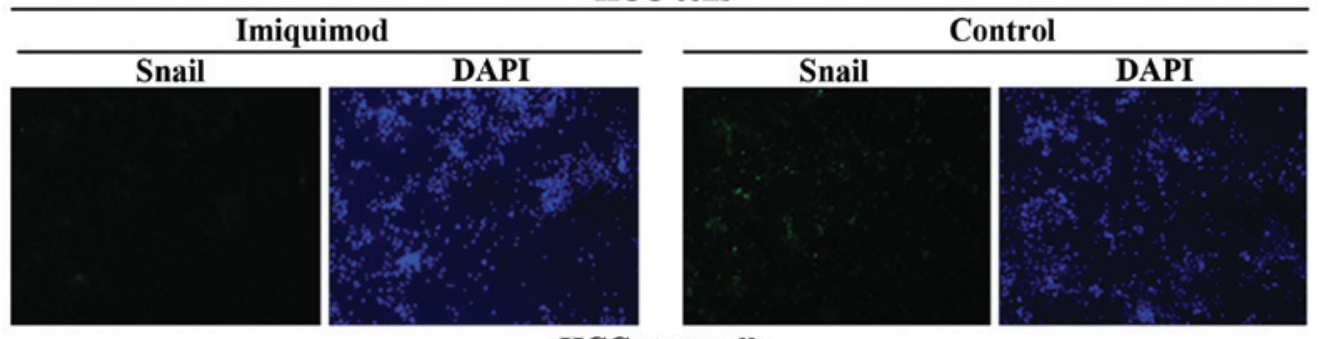

HCC stem cells
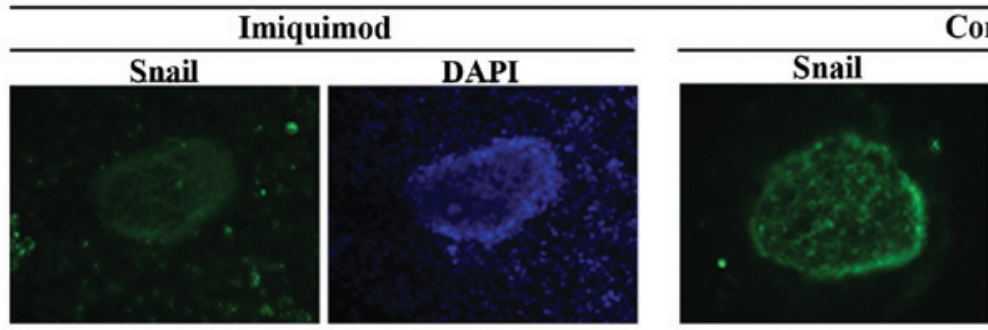

Control

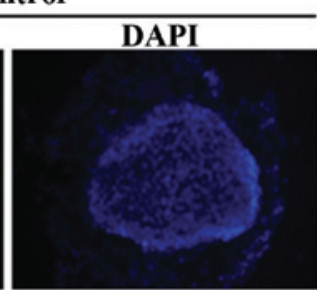

Figure 3. Suppression and associated mechanisms of Imiquimod on HCC stem cells. Signaling pathways implicated in TLR7 agonist induced cell repression were also evaluated. (A) Imiquimod effectively decreased ALDH ${ }^{+}$cells in the two $\mathrm{HCC}$ cell lines, and also decreased TLR7 ligand-induced stem cell number, $\mathrm{P}<0.01$. (B) Imiquimod treatment decreased IKK, NF- $\kappa \mathrm{B}$ and IL6 expression, which are all downstream genes of TLR7 signaling, indicating that traditional TLR7-IKK-NF-кB-IL6 signaling likely functioned in HCC cells. (C) The results of Snail staining in the various groups of HCC cells and HCC cancer stem cells revealed that Imiquimod treatment decreased Snail expression. Snail signal was stronger in mammospheres than that in cancer cells (magnification, HCC

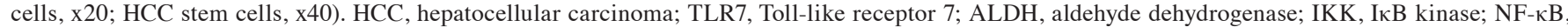
nuclear factor- $\kappa \mathrm{B}$; IL6, interleukin-6.

available therapies is required in order to reduce this risk. Understanding the mechanisms underlying hepatocarcinogenesis is vital for the development of more effective treatments. Chronic liver diseases, including chronic hepatitis and liver cirrhosis, are predisposing factors for the development of HCC. TLRs have a crucial role in conferring immunity against microbial pathogens and evidence has confirmed that TLRs are implicated in the pathogenesis of chronic liver disease, including 
that of HCC. TLR7 was previously confirmed to be upregulated in HCC tissues (20), and TLR7 stimulation significantly increased the proliferation of $\mathrm{HuH} 7$ cells, while inhibition of TLR7 using chloroquine resulted in significant inhibition (21). In vivo experiments revealed that TLR7 inhibition using chloroquine reduced tumor growth in xenograft models, and chloroquine also decreased liver fibrosis and tumor growth in the diethylnitrosamine and N-nitrosomorpholine model (22). However, how the TLR7 agonist Imiquimod functions in HCC cells, particularly in stem cells, has remained elusive. The putative mechanisms underlying Imiquimod functions require further investigation, which will aid the development of potential novel therapeutic strategies using the TLR7 agonists for the prevention of progression of primary liver cancers in susceptible patients, together with the results of other groups (23). CSCs were demonstrated to be crucial in the eradication of the tumor group entirely; therefore, the suppressive effects of TLR agonists on HCC cells and CSCs will have significance in the treatment of HCC, in particular, the TLR7 agonist Imiquimod $(9,23)$.

In the present study, the proliferative inhibition of Imiquimod on HCC cell lines was first evaluated, and inhibitory effects of Imiquimod were observed in the two HCC cell lines. Following confirmation of the appropriate and effective concentration of Imiquimod, it was demonstrated that Imiquimod treatment attenuated TLR7 ligand-induced cancer cell proliferation. To explore the functions of Imiquimod on HCC stem cells, the cells were treated with Imiquimod for $48 \mathrm{~h}$, and the self-renewal ability was determined using mammosphere formation assay. Imiquimod (100 $\mu \mathrm{l})$ significantly inhibited the self-renewal of HCC stem cells. Flow cytometry-based ALDEFLUOR fluorescent sorting, which aims to separate $\mathrm{ALDH}^{+}$cells, also demonstrated the influence of Imiquimod on stem cell numbers. For the first time, to the best of our knowledge, the present results proved that TLR7 agonist Imiquimod was able to suppress HCC cell and stem cell proliferation and self-renewal, an effect which may be mediated by TLR7-IKK-NFKB-IL6 signaling. The systemic administration of the selective TLR7 agonist resulted in dose-dependent changes in HCC cells, indicating the potential for novel therapeutic strategies for the prevention and progression of primary liver through targeting CSC groups. Further research is required to analyze the effects of additional TLR7 agonists in the inhibition of hepatic carcinoma by suppressing the ratios of CSCs and to elucidate their associated mechanisms. In addition, further in vivo experiments are required, to confirm the efficacy and safety of TLR7 agonist-based anticancer therapies.

\section{References}

1. Jemal A, Bray F, Center MM, Ferlay J, Ward E and Forman D: Global cancer statistics. CA Cancer J Clin 61: 69-90, 2011.

2. Thun MJ, DeLancey JO, Center MM, Jemal A and Ward EM: The global burden of cancer: Priorities for prevention. Carcinogenesis 31: 100-110, 2010.

3. Ferlay J, Shin H-R, Bray F, Forman D, Mathers C and Parkin DM: Estimates of worldwide burden of cancer in 2008: GLOBOCAN 2008. Int J Cancer 127: 2893-2917, 2010.

4. Wu HC and Santella R: The role of aflatoxins in hepatocellular carcinoma. Hepat Mon 12: e7238, 2012.
5. Dennison U, McKernan DP, Scully P, Clarke G, Cryan J and Dinan T: Menstrual cycle influences toll-like receptor responses. Neuroimmunomodulation 19: 171-179, 2012.

6. Kesar V and Odin JA: Toll-like receptors and liver disease. Liver Int 34: 184-196, 2014.

7. Mencin A, Kluwe J and Schwabe RF: Toll-like receptors as targets in chronic liver diseases. Gut 58: 704-720, 2009.

8. Mittal D, Saccheri F, Vénéreau E, Pusterla T, Bianchi ME and Rescigno M: TLR4-mediated skin carcinogenesis is dependent on immune and radioresistant cells. EMBO J 29: 2242-2252, 2010.

9. Dapito DH, Mencin A, Gwak GY, Pradere JP, Jang MK, Mederacke I, Caviglia JM, Khiabanian H, Adeyemi A, Bataller R, et al: Promotion of Hepatocellular Carcinoma by the Intestinal Microbiota and TLR4. Cancer Cell 21: 504-516, 2012.

10. Ji R, Tian S, Lu HJ, Lu Q, Zheng Y, Wang X, Ding J, Li Q and $\mathrm{Lu} \mathrm{Q}$ : TAM receptors affect adult brain neurogenesis by negative regulation of microglial cell activation. J Immunol 191: 6165-6177, 2013.

11. Larangé A, Antonios D, Pallardy M and Kerdine-Römer S: TLR7 and TLR8 agonists trigger different signaling pathways for human dendritic cell maturation. J Leukoc Biol 85: 673-683, 2009.

12. Wang Y, Abel K, Lantz K, Krieg AM, McChesney MB and Miller CJ: The Toll-like receptor 7 (TLR7) agonist, Imiquimod and the TLR9 agonist, CpG ODN, induce antiviral cytokines and chemokines but do not prevent vaginal transmission of simian immunodeficiency virus when applied intravaginally to rhesus macaques. J Virol 79: 14355-14370, 2005.

13. Oosterhoff D, Heusinkveld M, Lougheed SM, Kosten I, Lindstedt M, Bruijns SC, van Es T, van Kooyk Y, van der Burg SH and de Gruijl TD: Intradermal delivery of TLR agonists in a human explant skin model: Preferential activation of migratory dendritic cells by polyribosinic-polyribocytidylic acid and peptidoglycans. J Immunol 190: 3338-3345, 2013.

14. O'Neill LA, Bryant CE and Doyle SL: Therapeutic targeting of Toll-like receptors for infectious and inflammatory diseases and cancer. Pharmacol Rev 61: 177-197, 2009.

15. Sun X, Jiao X, Pestell TG, Fan C, Qin S, Mirabelli E, Ren H and Pestell RG: MicroRNAs and cancer stem cells: The sword and the shield. Oncogene 33: 4967-4977, 2014.

16. Kreso A and Dick JE: Evolution of the cancer stem cell model. Cell Stem Cell 14: 275-291, 2014

17. Sun X, Qin S, Fan C, Xu C, Du N and Ren H: Let-7: A regulator of the ER $\alpha$ signaling pathway in human breast tumors and breast cancer stem cells. Oncol Rep 29: 2079-2087, 2013.

18. Cicalese A, Bonizzi G, Pasi CE, Faretta M, Ronzoni S, Giulini B, Brisken C, Minucci S, Di Fiore PP and Pelicci PG: The tumor suppressor 553 regulates polarity of self-renewing divisions in mammary stem cells. Cell 138: 1083-1095, 2009.

19. Abdel-Raouf TA, Ahmed A, Zaki WK, Abdella HM and Zid MA: Study of toll-like receptor 7 expression and interferon $\alpha$ in Egyptian patients with chronic hepatitis $\mathrm{C}$ infection and hepatocellular carcinoma. Egypt J Med Hum Genet 15: 387-392, 2014.

20. Mohamed FE, Al-Jehani RM, Minogue SS, Andreola F, Winstanley A, Olde Damink SW, Habtesion A, Malagó M, Davies N, Luong TV, et al: Effect of toll-like receptor 7 and 9 targeted therapy to prevent the development of hepatocellular carcinoma. Liver Int 35: 1063-1076, 2015.

21. Guiducci C, Tripodo C, Gong M, Sangaletti S, Colombo MP, Coffman RL and Barrat FJ: Autoimmune skin inflammation is dependent on plasmacytoid dendritic cell activation by nucleic acids via TLR7 and TLR9. J Exp Med 207: 2931-2942, 2010.

22. Tazzyman S, Barry ST, Ashton S, Wood P, Blakey D, Lewis CE and Murdoch C: Inhibition of neutrophil infiltration into A549 lung tumors in vitro and in vivo using a CXCR2-specific antagonist is associated with reduced tumor growth. Int $\mathbf{J}$ Cancer 129: 847-858, 2011.

23. Adams S, Kozhaya L, Martiniuk F, Meng TC, Chiriboga L, Liebes L, Hochman T, Shuman N, Axelrod D, Speyer J, et al: Topical TLR7 agonist imiquimod can induce immune-mediated rejection of skin metastases in patients with breast cancer. Clin Cancer Res 18: 6748-6757, 2012. 